\title{
DEVENIR DES MÉTAUX LOURDS (CUIVRE ET NICKEL) DANS LES DÉCHARGES D'ORDURES MÉNAGÈRES
}

\author{
Thomas Lagier, Geneviève Feuillade et Guy Matejka \\ Laboratoire des sciences de l'eau et de l'environnement, École nationale supérieure d'ingénieurs de Limoges
}

L'objectif de ce travail est d'étudier les processus susceptibles d'augmenter la solubilité des métaux lourds piégés sous des formes réduites dans les décharges d'ordures ménagères (en particulier formes sulfures). Trois mécanismes ont été étudiés en réacteur discontinu: l'oxydation des phases sulfures à l'air, la complexation de métaux à la matière organique soluble du lixiviat et le changement du pH du milieu. Les essais menés en laboratoire à partir de phases solides synthétiques de sulfures de fer et sur deux métaux (nickel et cuivre) indiquent que ces derniers se comportent de façon très différente. Le cuivre, très insoluble en milieu réduit, n'est solubilisé que pour une oxydation quasi complète du milieu et pour des $\mathrm{pH}$ bas. Le nickel se solubilise dés le début de l'oxydation du milieu et pour des $\mathrm{pH}$ proches de la neutralité. L'oxydation influence la solubilisation de ces métaux mais l'acidification du milieu comme la complexation peuvent également contribuer à augmenter de façon significative la solubilisation de ces deux métaux. Les résultats apportent des éclairages sur les mécanismes susceptibles de se produire dans des décharges à long terme ou au cours d'opérations de réhabilitation.

This paper deals with main mechanisms which are likely to increase the solubility of heavy metal trapped under reduced forms (sulfur species) in landfills. Three mechanisms were studied under batch conditions: air induced sulfur species oxidation, complexation of metals to the soluble organic matter of leachate and $\mathrm{pH}$ range variation. The tests carried out in laboratory upon synthetic solid sulfur phases of iron and of two metals (nickel and copper) indicate that the latter behavior is very different. Copper, the solubility of which under reduced conditions is low, is solubilized only under complete oxidation conditions and at low pH. Nickel is solubilized as soon as oxidation starts and at $\mathrm{pH}$ close to neutral values. Oxidation influences these metal solubilization but acidification and complexation can also contribute to increase at a significant level these two metals solubilization. These results show that these mechanisms are likely to occur in long-term landfills or when rehabilitation operations are proceeded.

\section{PROBLEMATIQUE}

Pendant de nombreuses années, la mise en décharge a constitué la principale voie d'élimination des déchets. De grandes quantités de métaux lourds ont ainsi été enfouies au cours du stockage des déchets urbains ou industriels. Les métaux lourds sont présents dans la plupart des constituants des déchets (Rousseau et al., 1990). Les lixiviats constituent le principal vecteur de transport de la pollution métallique. Même si, dans les centres d'enfouissement technique récents, cet effluent est particulièrement contrôlé (décret du 7 septembre 1997), les décharges anciennes, continueront à produire des lixiviats pendant plusieurs dizaines d'années après leur fermeture, participant ainsi à la pollution du milieu naturel et potentiellement à la migration des métaux lourds dans l'environnement.

Les teneurs en métaux lourds mesurées dans les lixiviats sont toujours faibles pendant les périodes d'exploitation de la décharge (Robinson, 1995; Flyhammar, 1997), bien que la matière organique et minérale du lixiviat ait tendance à accroître la mobilité des métaux en les complexant (Christensen et al., 1996).

Les métaux, très peu mobiles, sont donc majoritairement piégés dans les décharges (Revans et al., 1999; Flyhammar et Hakansson, 1999; Rinke, 1999). Baccini et al. (1987) estiment que plus de $99,9 \%$ du stock de métaux lourds est encore piégé dans la décharge au bout de 30 ans. La faible solubilisation des métaux lourds peut être expliquée par les formes sous lesquelles ils se trouvent au sein de la décharge. En effet, l'ensemble des études concernant la spéciation chimique des métaux au sein de déchets âgées permet de conclure que les métaux remobilisables sont associés aux sulfures et à la matière organique dans les décharges (Rouyer, 1990; Del Fava, 1992; Legret, 1993; Flyhammar et Hakansson, 1999). Le piégeage chimique crée des réservoirs où se concentrent les métaux lourds, leur solubilité dans le lixiviat est faible (Christensen et al., 1994; Aulin et Neretnieks, 1995; Bozkurt et al., 1999). Ces métaux sont potentiellement mobilisables en cas de changement de conditions. Les processus identifiés dans la bibliographie comme étant susceptibles d'augmenter la mobilité des métaux sont les suivants: 
- diminution du pH consécutive à l'oxydation ou à des facteurs extérieurs (pluies acides...),

$\equiv \mathrm{S}-\mathrm{M}+\mathrm{xH}^{+}=\equiv \mathrm{S}-\mathrm{H}_{\mathrm{x}}+\mathrm{M}^{\mathrm{x}}$

avec $\equiv \mathrm{S}$ : solide de surface

- oxydation des zones anaérobies de la décharge qui conduit à remettre en solution des métaux piégés sous des formes oxydables (sulfures et matière organique),

$\mathrm{MS}+2 \mathrm{O}_{2}=\mathrm{M}^{2+}+\mathrm{SO}_{4}^{2-}$

avec MS: sulfures de métaux

- complexation des métaux par la matière organique et minérale du lixiviat.

$\equiv S-M+M O_{(s)}=\equiv S+M O-M_{(s)}$

avec $M O_{(s)}$ : matière organique soluble du lixiviat

Ces différents processus peuvent être liés. S'il est certain qu'ils jouent un rôle clef dans la solubilité des métaux, leur influence réelle est difficilement quantifiable et la littérature n'est pas toujours unanime.

\section{MATÉRIELS ET MÉTHODES}

\section{Choix des métaux}

Les métaux lourds choisis sont le cuivre et le nickel qui sont présents dans les ordures ménagères. Le cuivre peut être considéré comme métal de référence car il est très fréquemment utilisé dans les travaux sur la complexation. Le nickel a été choisi pour ses propriétés différentes de celles du cuivre et plus particulièrement pour sa solubilité, relativement forte, en présence de sulfures ( $\mathrm{pK}$ s de $\mathrm{NiS}=23,8$ et $\mathrm{pKs}$ de $\mathrm{CuS}=35, \mathrm{I})$. En présence à la fois de matière organique et de sulfures, ce métal apparaît comme le plus mobile et il peut se trouver en grande quantité dans les panaches de lixiviats sous les décharges comme l'indiquent Warwick et al. (1994) et Altmann et Bourg (1997).

\section{Choix de la phase solide dans laquelle sont piégés les métaux lourds}

Les phases solides ont été synthétisées à partir du protocole proposé par Puginier (1995) et repris plus récemment par Altmann et Bourg (1997). Ce protocole permet de simuler les phases sous lesquelles se trouvent les métaux potentiellement remobilisables dans une décharge (phase de sulfures de fer et de métaux).

\section{Choix de la matière organique}

Les phénomènes de complexation matière organique métaux lourds sont de toute première importance dans les lixiviats. Warwick et al., 1994; Christensen et al., 1996; Majone et al., 1996 mettent en évidence que la matière organique de haut poids moléculaire (type acides humiques et fulviques) joue un rôle prédominant dans les lixiviats. Les substances humiques sont d'ailleurs bien connues et étudiées dans d'autres compartiments environnementaux et de nombreux auteurs ont pu montrer leurs importantes capacités de complexation vis-à-vis des métaux (Weis et al., 1989; Christensen et al., 1998).
Afin d'étudier les mécanismes de complexation de la matière organique avec les métaux lourds, les acides humiques et fulviques du lixiviat ont donc été extraits du lixiviat par un protocole proche de celui préconisé par l'IHSS (International Humic Substances Society). Ces molécules ont ensuite été caractérisées par différentes techniques analytiques (analyses élémentaire, spectrofluorescence, UV-visible, IRTF, RMN ${ }^{13} \mathrm{C}$, potentiométrie...) (Lagier et al., 2000a).

Leurs propriétés complexantes avec le cuivre ont été étudiées et ont permis de déterminer des constantes conditionnelles de complexation de l'ordre de $1.10^{5}$ à $2.10^{6}$ (Lagier et al., 2000b).

Les expériences ont été effectuées en réacteur (figure I) afin d'étudier: i) l'influence des macromolécules extraites du lixiviat; ii) l'influence de l'aération et iii) l'influence du $\mathrm{pH}$ sur la solubilisation du cuivre et du nickel.

Le dispositif expérimental permet la mesure et la régulation du $\mathrm{pH}$, du potentiel redox et de la température. Des prélèvements de suspension sont également effectués. Les concentrations en carbone organique dissous (COD) en

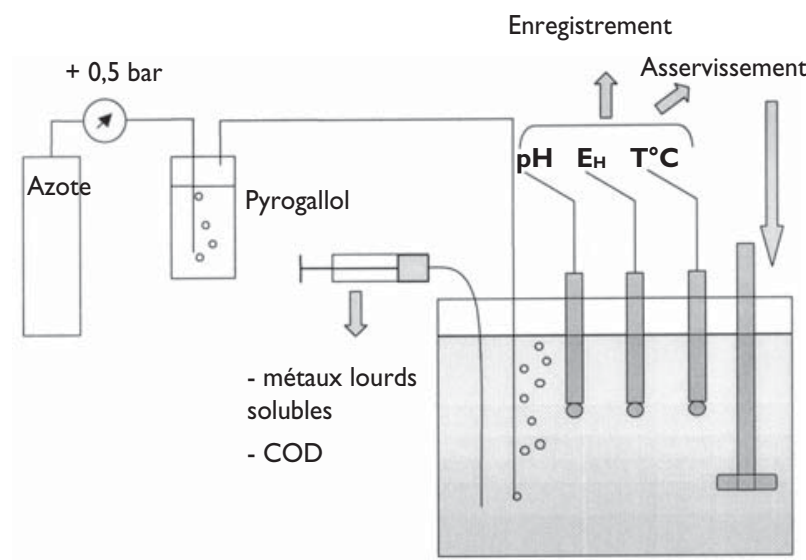

Figure I : Montage expérimental pour l'étude en réacteur

nickel et en cuivre en solution sont mesurées après filtration de l'échantillon $(0,45 \mu \mathrm{m})$. La suspension est appelée pulpe.

Les conditions expérimentales sont présentées dans le tableau I; la force ionique de la suspension est fixée à $0, \mathrm{I} \mathrm{mol} . \mathrm{L}^{-1}\left(\mathrm{NaNO}_{3}\right)$ qui correspond à l'ordre de grandeur de la force ionique d'un lixiviat. La température est fixe $\left(25^{\circ} \mathrm{C}\right)$, la concentration en cuivre et en nickel est de 90 umol. $\mathrm{L}^{-1}$, ces métaux sont piégés (co-précipités et adsorbés) sur des sulfures de fer.

Le $\mathrm{pH}$ est dans certains essais régulé par ajout de $\mathrm{NaOH}$ ou de $\mathrm{HNO}_{3}$ concentré.

\section{Tableau I : Conditions expérimentales}

\begin{tabular}{|c|c|c|c|c|c|c|c|c|}
\hline $\begin{array}{c}\text { État } \\
\text { d'oxydation }\end{array}$ & \multicolumn{3}{|c|}{$\begin{array}{l}\text { Pulpe } \\
\text { réduite }\end{array}$} & \multicolumn{2}{|c|}{ Oxydation } & \multicolumn{3}{|c|}{$\begin{array}{c}\text { Pulpe } \\
\text { oxydée }\end{array}$} \\
\hline $\mathrm{pH}$ & 2 & 7 & 12 & 7 & libre & 2 & 7 & 12 \\
\hline COD & \multicolumn{8}{|c|}{ entre 0 et $200 \mathrm{mg}$} \\
\hline
\end{tabular}




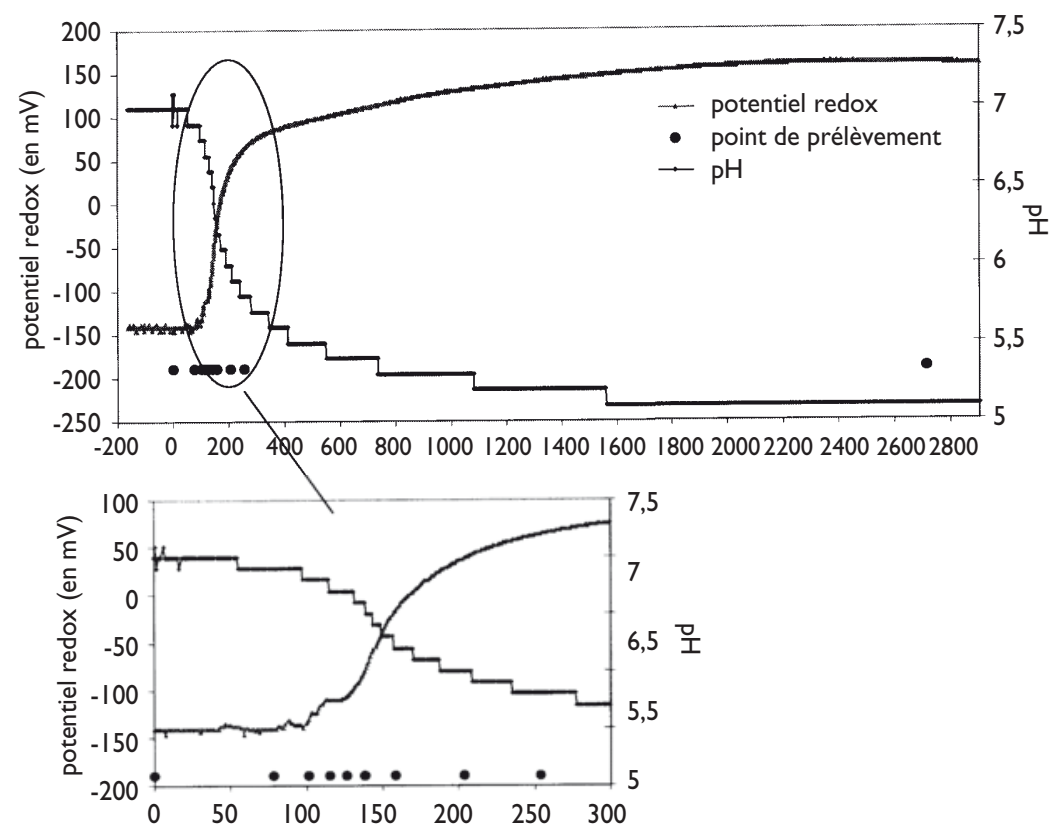

Figure 2: Évolution du pH et du potentiel redox de la pulpe au cours de l'oxydation; ouverture à l'air à $t_{0}$ après ([COD] initial $=50 \mathrm{mgC}^{\left.\mathrm{L}^{-1}\right)}$

moins de 7 heures.

Les prélèvements sont effectués à intervalle de potentiel redox régulier. Les résultats des dosages du carbone organique, du nickel et du cuivre en solution sont présentés figure 3.

La teneur en COD a tendance à diminuer légèrement (de 90 à $75 \%$ ) au cours de l'oxydation. Une partie du carbone organique dissous introduit est donc retenue dans la phase solide. II peut être associé à des formes hydroxydes métalliques ou bien précipité suite à la diminution $\mathrm{du} \mathrm{pH}$ du milieu.

Le nickel et le cuivre se comportent de manières différentes. Alors que le nickel se solubilise dés l'ouverture à l'air du réacteur et le début d'oxydation des phases sulfures, le cuivre reste très insoluble sur la majeure partie de l'essai et ce n'est que pour des conditions beaucoup plus oxydantes et lorsque le $\mathrm{pH}$ a diminué de façon significative que le cuivre se retrouve dans la phase soluble. En fin

L'étude des phénomènes d'oxydation est effectuée en aérant à l'air la suspension réduite.

\section{RÉSULTATS ET DISCUSSION}

\section{Influence de l'oxydation}

Le réacteur contenant la pulpe réduite (figure I) est ouvert. La mise à l'air entraîne alors une oxydation des phases sulfures; l'essai présenté (figure 2 et 3 ) a été effectué en présence de matière organique $\left(50 \mathrm{mgC} . \mathrm{L}^{-1}\right)$ et sans régulation $\mathrm{du} \mathrm{pH}$.

Après ouverture à l'air du réacteur, le potentiel redox passe rapidement d'une valeur de $-150 \mathrm{mV}$ à des valeurs de potentiel redox positives et atteint en fin d'essai $150 \mathrm{mV}$. L'oxydation à l'air des espèces du soufre conduit également à une chute du $\mathrm{pH}$ simultanément à l'augmentation du potentiel. Le $\mathrm{pH}$ passe d'une valeur de 7,3 à moins de 5,5 en

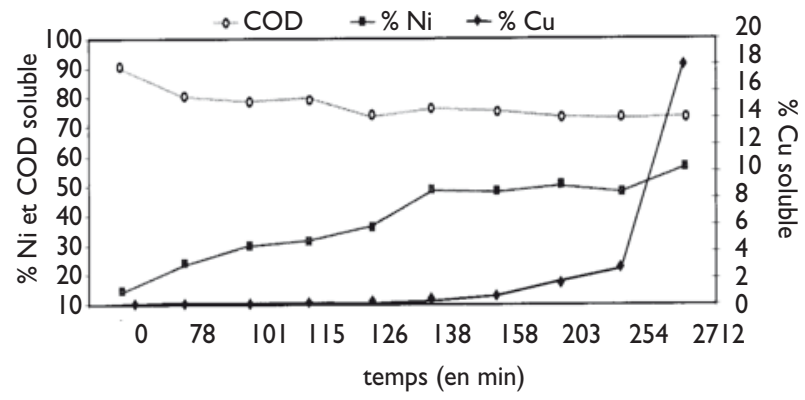

Figure 3: Suivi du COD, du nickel et du cuivre soluble en fonction de l'état d'oxydation de la pulpe d'essai la teneur en cuivre en solution atteint $18 \%$, celle du nickel dépasse $50 \%$ moins de $3 \mathrm{~h}$ après le début de l'oxydation.

\section{Influence de la matière \\ organique}

L'influence de la concentration en carbone organique dissous (macromolécules complexantes) peut être mise en évidence (figure 4). En effet, on observe sur les quatre courbes une tendance à l'augmentation de la quantité de métaux en solution lorsque la teneur en COD du milieu augmente. Cette augmentation est très significative dans la pulpe oxydée, résultat d'autant plus marqué pour le nickel dont la concentration en solution passe de 24 à $76 \%$ entre 0 et $200 \mathrm{mgC}$. $\mathrm{L}^{-1}$. Les métaux piégés dans la pulpe réduite restent relativement insolubles quelle que soit la teneur en

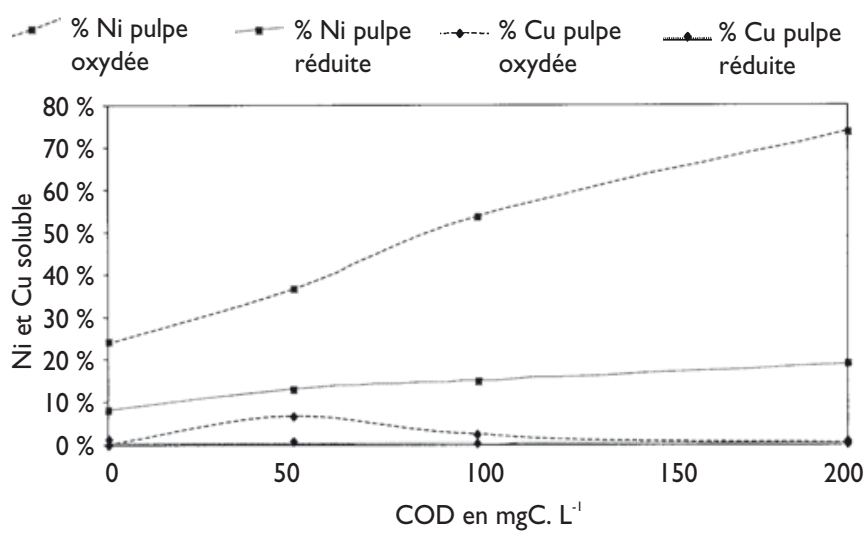

Figure 4: Proportion de cuivre et de nickel en solution en fonction de la concentration en COD : pulpe en conditions réduites puis oxydée à l'air $(\mathrm{pH} 7)$ 
COD et les variations observées ne sont pas significatives.

On observe également que la quantité de métal en solution est très variable: entre 10 et $74 \%$ pour le nickel selon les conditions expérimentales et moins de $6 \%$ pour le cuivre. En conditions réduites le cuivre est jusqu'à 80 fois moins soluble que le nickel, ce résultat s'explique par les différences importantes de solubilité des sulfures de ces deux métaux.

\section{Influence du pH}

Les essais présentés (figure 5) ont été effectués entre $\mathrm{pH} 2$ et 12 sur la suspension réduite (pulpe réduite) puis sur la suspension après une phase d'oxydation à l'air de 24 h (pulpe oxydée). La concentration en matière organique est nulle. Les comportements du cuivre et du nickel sont très différents selon, l'état d'oxydation de la pulpe. Dans le cas de la pulpe réduite (a), la diminution du $\mathrm{pH}$ entraîne une solubilisation importante du nickel qui se trouve totalement soluble vers $\mathrm{pH}$ 2. En revanche, le cuivre reste relativement peu soluble quel que soit le $\mathrm{pH}$ du milieu.

Si la pulpe est oxydée (b), le cuivre comme le nickel se solubilisent lorsque le $\mathrm{pH}$ diminue, la courbe obtenue est caractéristique des échanges de surface de type adsorption (Boudou et Ribeyre, 1989; Stumm et Morgan, 1995).
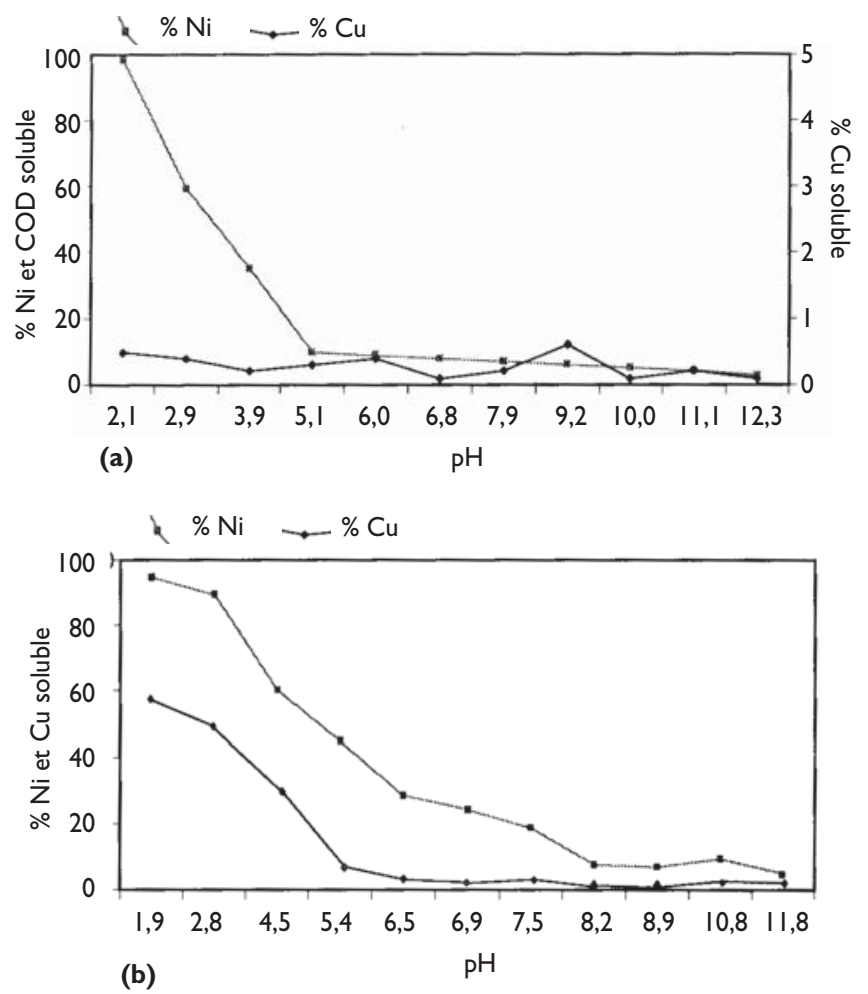

Figure 5 : Proportion de cuivre et de nickel en solution en fonction du $\mathrm{pH}$ : pulpe en conditions réduites (a) puis oxydée à l'air (b) (COD nul)
Tableau 2: Solubilisation du cuivre et du nickel sous forme de sulfures en fonction des conditions expérimentales

$\begin{array}{clll}\begin{array}{c}\text { Cas I : pas } \\ \text { d'acidification } \\ \text { du milieu }\end{array} & \begin{array}{c}\text { Cas 2: } \\ \text { acidification } \\ \text { du milieu }\end{array} & \begin{array}{c}\text { Cas 3: pas } \\ \text { d'acidification } \\ \text { du milieu }\end{array} & \begin{array}{c}\text { Cas 4: } \\ \text { acidification } \\ \text { du milieu }\end{array} \\ \begin{array}{c}\text { Scu très faible } \\ \text { ôle du COD }\end{array} & \begin{array}{l}\mathrm{S}_{\mathrm{Cu}} \text { faible } \\ \text { Scu augmente }\end{array} & \begin{array}{c}\text { SCu moyenne } \\ \text { rôle du COD }\end{array} & \begin{array}{l}\text { Scu augmente } \\ \text { fortement }\end{array} \\ \text { mal déterminé } & \text { légèrement avec COD } & \text { mal déterminé } & S_{C u} \text { diminue avec COD } \\ \mathrm{S}_{\mathrm{Ni}} \text { faible } & \mathrm{S}_{\mathrm{Ni}} \text { augmente } & \mathrm{S}_{\mathrm{Ni}} \text { moyenne } & \mathrm{S}_{\mathrm{Ni}} \text { augmente } \\ \mathrm{S}_{\mathrm{Ni}} \text { augmente } & \mathrm{S}_{\mathrm{Ni}} \text { augmente } \mathrm{S} & \mathrm{Ni} \text { augmente } & \text { fortement } \\ \text { vec COD } & \text { avec COD } & \text { avec COD } & \mathrm{S}_{\mathrm{Ni}} \text { diminue avec COD }\end{array}$

\section{SYNTHÈSE ET CONCLUSION}

L'analyse des résultats obtenus à l'échelle laboratoire permet de dégager, en fonction des conditions du milieu, des tendances générales de comportement qui peuvent être transposées à la décharge d'ordures ménagères même s'il faut prendre des précautions importantes.

Le tableau 2 montre les principales conséquences sur la solubilisation des métaux d'une acidification du milieu, d'une oxydation à l'air ou de la complexation par la matière organique. Les métaux sont piégés sous des formes relativement stables en conditions anaérobies: sous leurs formes sulfures les métaux lourds sont très insolubles dans le lixiviat et ne peuvent donc pas migrer hors de la décharge. L'acidification, même importante, ne conduit pas à une augmentation de la concentration du cuivre en solution, seul le nickel est redissous pour des $\mathrm{pH}$ inférieurs à 5 . La complexation à la matière organique augmente la solubilité des métaux de façon non

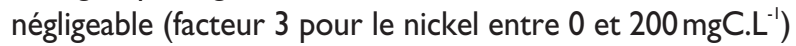
mais ces concentrations restent faibles.

En cas d'oxydation des déchets (au cours d'une réhabilitation du site ou à long terme), le risque de relargage de métaux par la décharge devient important. À pH 7, la solubilité du cuivre passe de valeurs inférieures à $\mathrm{I} \%$ à environ $20 \%$, celle du nickel passe de moins de $20 \%$ à plus de $70 \%$. Entre la pulpe réduite et oxydée, la diminution du $\mathrm{pH}$ (qui est d'ailleurs liée au processus d'oxydation) entraîne une solubilisation importante des métaux lourds.

Cette étude a permis d'observer qualitativement et quantitativement les effets de l'oxydation à l'air, de la complexation avec la matière organique ainsi que de la diminution du $\mathrm{pH}$ sur la solubilisation du cuivre et du nickel piégés sous des formes sulfures. Les résultats obtenus en laboratoire permettent d'appréhender les mécanismes qui peuvent se produire dans une décharge en distinguant plusieurs cas de figures selon que le milieu est oxydé ou réduit, tamponné ou non et selon qu'il y ait ou non présence de la matière organique. En cas de contact avec l'air de déchets stabilisés, l'oxydation des phases sulfures entraînerait une remobilisation massive des métaux aggravée par une diminution du $\mathrm{pH}$.

*Thomas Lagier, Geneviève Feuillade et Guy Matejka Laboratoire des sciences de l'eau et de l'environnement - École nationale supérieure d'ingénieurs de Limoges - 16, rue Atlantis - Parc Ester Technopole - 87068 Limoges 


\section{Bibliographie}

- Altmann R.S. et Bourg A.C.M., (1997): Cadmium mobilization under conditions simulating anaerobic to aerobic transition in a landfill leachate-polluted aquifer. Water Air Soil Poll., 94, 385-392.

-Aulin C. et Neretnieks I., (1995): A Material Balance for an Industrial Landfill, In proceeding Sardinia 95, Fifth International Waste Management and Landfill Symposium, eds. Christensen T.H. et al., Cagliari, vol. 3, I73-180.

- Baccini P., Henseler G., Figi R. et Belevi H., (1987): Water and element balances of municipal solid waste landfills. Waste Manage. Res. 5, 483-499.

- Boudou A. et Ribeyre F., (1989): Aquatic ecotoxicology: fundamental concepts and methodologies, volume I, CRC press. Boca Raton, Florida. 319 pages.

- Bozkurt S., Moreno L. et Neretnieks I., (1999): Long-term fate of organics in waste deposits and its effect on metal release. Sci. Total Environ., 228, I35-152

- Christensen T.H., Kjeldsen P., Albrechtsen H.J., Bjerg P.L. et Holm P.E (1994): Attenuation in landfill leachate pollutants in aquifers. Critical Reviews in Environ. Sci. Technol., 24, I I9-202.

- Christensen J.B., Jensen D.L. et Christensen T.H., (1996): Effect of dissolved organic carbon on the mobility of cadmium, nickel and zinc in leachate polluted groundwater. Wat. Res., 30, 3037-3049.

- Christensen J.B., Jensen D.L., Gron C. Filip Z. et Christensen T. H., (1998) Characterization of the dissolved organic carbon in landfill leachate polluted groundwater. Wat. Res., 32, 125-135.

- Del Fava J., (1992): Spéciation des métaux lourds dans les ordures ménagères et leur produit de traitement par digestion anaérobie. Thèse de doctorat, Université de Montpellier II.

- Flyhammar P., (1997): Estimation of heavy metal transformations in municipal solid waste. Sci. Tot. Environ., 198, I23 133.

- Flyhammar P. et Hakansson K., (I999) The mobilization of heavy metals in partly stabilised MSW during oxidation. In proceeding Sardinia 99, Seventh Internationa Waste Management and Landfill Symposium, eds. Christensen T.H. et al. Cagliari, Italy, 4-8 October, vol. I, 2I3218

- Lagier T., Feuillade G. et Matejka G. (2000) a: Characterisation and comparison of humic and fulvic like acids extracted from sanitary landfill leachate. accepté à Environ. Sci. pollut. Res.

- Lagier T., Feuillade G. et Matejka G. (2000) b: Interactions between copper orga- nic macromolecules: determination of conditional complexation constants. Agronomy, 20, 537-546.

- Legret M., (1993): Speciation and mobility of heavy metal in a simulated landfill. Proceeding Heavy metal in the Environment, Toronto, september, 462 467.

- Majone M., Petrangeli-Papini M. et Rolle E., (1996): Heavy metal speciation in landfill leachates by exchange on chelex100 resin. Environ. Technol., 17, 587595.

- Puginier B., (1995): Facteurs géochimiques contrôlant l'atténuation des polluants métalliques dans les panaches de lixiviats issus des décharges. Thèse de doctorat. Université de Toulouse.

- Revans A., Ross D., Gregory B., Meadows M., Harries C. et Gronow I. (1999): Long term fate of heavy metals in landfill. In proceeding Sardinia 99, Seventh International Waste Management and Landfill Symposium, eds. Christensen T.H. et al., Cagliari, Italy, 4-8 October, vol. I, 199-206.

- Rinke M., (1999): Migration de la pollution minérale dans les sols sous les décharges d'ordures ménagères: étude de cas. Thèse de doctorat, Université de Limoges.

- Robinson H.D., (1995). A review of the composition of leachates from domestic wastes in landfill sites. Report $\mathrm{n}^{\circ}$ CWM/072/95 UK Department of the Environment, Waste Technical Division, London.

- Rousseau P., Navarro A. et Vermande P. (1990): Distribution des 7 principaux métaux lourds dans les constituants des ordures ménagères. Tribune de l'eau, 42, 17-25.

- Rouyer P., (1990): Spéciation et élimiation des métaux lourds dans les résidus de digestion anaérobie des déchets urbains. Thèse de doctorat, Université de Montpellier II.

- Stumm W. et Morgan J. J., (1995)

Aquatic chemistry; Chemical Equilibria and Rates in Natural Waters. $3^{\text {rd }}$ edition. Eds John Wiley \& Sons

- Warwick P. Anderton W. Smith B. et Williams G.M., (1994): Nickel speciation in polluted groundwater at Villa Farm. Report of the European Commission EUR 14969 EN.

-Weis M., Abbt-Braun G. et Frimme F.H., (1989): Humic-like substances from landfill leachates-characterization and comparison with terrestrial and aquatic humic substances. Sci. Tot. Environ., 81/82, 343352.

\section{Bon de commande de l'ouvrage}

A retourner à SAP -7 , chemin de Gordes 38100 Grenoble - Tél. : 0476432864

Fax : 0476569409 - Mél : sap@pro-environnement.com avec votre réglement

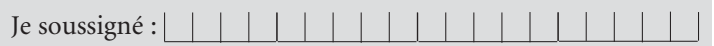

Entreprise : $\lfloor|||\downarrow||||+||||||||| \mid$

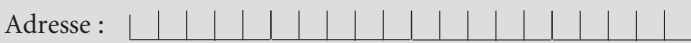

Code postal : $\lfloor||||\rfloor$ Ville : $|\perp||||L||| \mid$

déclare passer commande de.............. exemplaire(s) de l'ouvrage Décharges :quel avenir ? au prix unitaire de 125 F TTC franco (118,49 F HT + TVA 5,5 \% : 6,51 F).

Réglement à la commande par chèque ci-joint $\square$ ou par virement $\square$ au CCP SAP 0134695R028

Grenoble. Je désire une facture en retour en exemplaire(s).

Signature ou cachet :

\section{DÉCHARGES : quel avenir ?}

\section{Gérard Bertolini}

Ce livre n'est pas un Traité technique, même si les considérations techniques $n^{\prime}$ en sont pas absentes. Car il y a la technique et le reste, qui ne saurait être négligé, les conceptions et les réalisations, les pratiques et les représentations, et leurs interactions. Les sciences de l'homme et de la société, jusqu'à la psychanalyse et la philosophie, ont leur mot à dire. Désignant à la fois un acte, un lieu (un

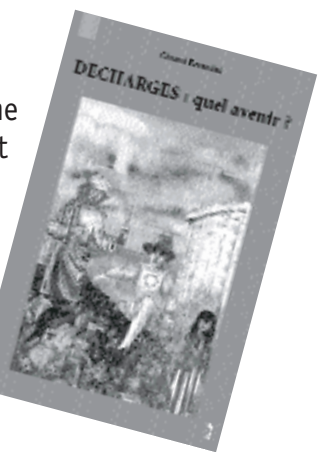
site) et un mode d'élimination des déchets, la décharge est un «fait social »; l'ambition est d'en réaliser l'anthropologie, de la saisir dans toutes ses dimensions et tous ses états. La recherche ne porte pas seulement sur les pratiques et les signifiants de la décharge. L'appréhension de son évolution à travers les âges, en privilégiant toutefois les changements les plus récents, est orientée par le souci d'une vision d'avenir ou, pour le moins, d'interrogations prospectives. À son tour, la décharge n'est-elle pas «un méga-objet périssable»? Ce livre n'est ni un plaidoyer pour, ni un plaidoyer contre la décharge. Sa part de subjectivité reflète bien sûr, outre les limites de ses connaissances, la sensibilité de son auteur.

Format : $15,5 * 24-108$ pages + couverture

Prix TTC franco : $125 \mathrm{~F}$ (Prix HT : 118,49 F - TVA 5,5\% : 6,51 F) 